\title{
RNA sequencing differential gene expression analysis of isolated perfused bovine udders experimentally inoculated with Streptococcus agalactiae
}

\author{
A. P. Sbardella, ${ }^{1}$ M. M. D. C. A. Weller, ${ }^{2}$ I. Fonseca, ${ }^{2}$ N. B. Stafuzza, ${ }^{1}$ P. A. Bernardes, ${ }^{1}$ F. F. e Silva, ${ }^{3}$ \\ M. V. G. B. da Silva, ${ }^{2}$ M. F. Martins, ${ }^{2}$ and D. P. Munari ${ }^{1 *}$ \\ ${ }^{1}$ Departamento de Ciências Exatas, Universidade Estadual Paulista-UNESP/FCAV, Jaboticabal 14884-900, Brazil \\ ${ }^{2}$ Empresa Brasileira de Pesquisa Agropecuária-Embrapa Gado de Leite, Juiz de Fora 36038-330, Brazil \\ ${ }^{3}$ Departamento de Zootecnia, Universidade Federal de Viçosa, Viçosa 36570-000, Brazil
}

\section{ABSTRACT}

The aim of this study was to elucidate the differential gene expression in the RNA sequencing transcriptome of isolated perfused udders collected from 4 slaughtered Holstein $\times$ Zebu crossbred dairy cows experimentally inoculated with Streptococcus agalactiae. We studied 3 different statistical tools (edgeR, baySeq, and Cuffdiff 2). In summary, 2 quarters of each udder were experimentally inoculated with Strep. agalactiae and the other 2 were used as a control. Mammary tissue biopsies were collected at times 0 and $3 \mathrm{~h}$ after infection. The total RNA was extracted and sequenced on an Illumina HiSeq 2000 (Illumina Inc., San Diego, CA). Transcripts were assembled from the reads aligned to the bovine UMD 3.1 reference genome, and the statistical analyses were performed using the previously mentioned tools (edgeR, baySeq, and Cuffdiff 2). Finally, the identified genes were submitted to pathway enrichment analysis. A total of 1,756, 1,161, and 3,389 genes with differential gene expression were identified when using edgeR, baySeq, and Cuffdiff 2, respectively. A total of 122 genes were identified by the overlapping of the 3 methods; however, only the platelet activation presented a significantly enriched pathway. From the results, we suggest the FCER1G, GNAI2, ORAI1, and VASP genes shared among the 3 methods in this pathway for posterior biological validation.

Key words: baySeq, crossbred dairy cow, Cuffdiff 2, edgeR

\section{INTRODUCTION}

The identification of genes expressed in different tissues leads to a better understanding of molecular mechanisms that are relevant for animal production.

Received August 8, 2018.

Accepted October 28, 2018.

*Corresponding author: danisio@fcav.unesp.br
Ribonucleic acid sequencing (RNA-seq) allows a wider gene expression analysis because the total RNA from a given tissue can be sequenced (Wang at al., 2009). Different statistical methods are available for performing RNA-seq differential gene expression analysis in a range of biological experiments (Hardcastle and Kelly, 2010; Robinson et al., 2010a).

The edgeR (Robinson et al., 2010a) and baySeq (Hardcastle and Kelly, 2010) packages from Bioconductor/R software and the Cuffdiff 2 (Trapnell et al., 2013) package implemented in Cufflinks software have been widely used to perform statistical analysis of RNA-seq data (Soneson and Delorenzi, 2013; Seyednasrollah et al., 2015). These tools were proposed to detect differential gene expression based on different statistical assumptions, leading to distinct results. Thus, the evaluation of all these methods in RNA-seq experimental designs is recommended (Seyednasrollah et al., 2015). This evaluation is used to improve the selection of candidate genes for posterior biological validation based on pathway enrichment analysis. The aim of this study was to elucidate the differential gene expression in the RNAseq transcriptome of isolated perfused udders, collected from Holstein $\times$ Zebu crossbred dairy cows experimentally inoculated with Streptococcus agalactiae, using the previously mentioned statistical methods.

\section{MATERIALS AND METHODS}

All animal care and handling procedures followed regulations approved by the animal research ethics committee of Embrapa Dairy Cattle (Juiz de Fora, MG, Brazil; license no. 11/2011).

\section{Data Collection}

The samples were obtained from 4 Holstein $\times$ Zebu crossbred dairy cows slaughtered in the experimental center "Campo Experimental José Henrique Bruschi" at Embrapa Dairy Cattle. Their udders were extracted 
and kept in an isolated perfused system. Two quarters of each udder were experimentally inoculated with Strep. agalactiae, and the other 2 quarters were used as a control. Mammary tissue biopsies were collected at times 0 and $3 \mathrm{~h}$ after infection.

\section{RNA Extraction}

The RNA extraction was performed in the Molecular Genetics Laboratory at Embrapa Dairy Cattle. The total RNA was extracted in 2 replicates using the RNeasy Mini Kit (Qiagen, Valencia, CA). The protocol is based on cell lysis, followed by a DNase treatment to avoid DNA contamination, binding of RNA to the silica gel membrane, purification, and subsequent elution of total RNA. The total isolated RNA was quantified by spectrophotometry (NanoDrop, Wilmington, DE), and its integrity was evaluated by RNA integrity number score on a Bioanalyzer 2100 (Agilent Technologies, Santa Clara, CA). The average RNA integrity number \pm standard deviation was $6.69 \pm 0.46$. For each udder, a pool of RNA sample from the 2 inoculated quarters was obtained and another pool of RNA sample was collected from the 2 control quarters. These collections were made 2 times $(0$ and $3 \mathrm{~h}$ ) for all 4 animals, totaling 16 samples ( 4 biological replicates $\times 2$ collections $\times 2$ conditions $)$.

\section{RNA Sequencing}

The RNA was sequenced by a commercial provider that prepared cDNA libraries using a TruSeq RNA sample preparation kit (Illumina, San Diego, CA), generating about 19 million 100-bp reads. The libraries were quantified by real-time PCR and sequenced $(2 \times$ 100 paired-end reads) on a HiSeq 2000 sequencer (Illumina) using the TruSeq SBS Kit version 3 (Illumina).

\section{Quality Control and Alignment}

Quality control was performed using FastQC (version 0.65; Afgan et al., 2016; https://usegalaxy.org/). Adapter sequences were removed from reads; however, low-quality or ambiguous reads were not trimmed be- cause a preliminary test showed that they did not align to the reference genome.

Reads were mapped to the bovine genome UMD 3.1 assembly (http://www.ncbi.nlm.nih.gov/genome?term = bos\%20taurus) using TopHat (version 2.1.0; Trapnell et al., 2009) with default settings and following the authors' protocol. The unmapped reads were subjected to a BLAST search on a nonredundant protein database using NCBI BLAST + blastx (version 0.1.07; Camacho et al., 2009).

Following the alignment, Cufflinks (version 2.2.1.0) with default settings (Trapnell et al., 2010) was used to assemble the transcripts. The abundance of the transcripts was reported by Cufflinks as fragments per kilobase of transcript per million fragments sequenced, which was the normalized expression level, later used by the Cuffdiff 2 .

Gene expression was then calculated by counting the reads mapped to exons using htseq-count (version 0.6.1; Anders et al., 2015). The normalized gene expression data were summarized in an expression matrix, which was later used by edgeR and baySeq. A multidimensional scaling method was used to represent the similarities between samples because it reproduces the observed distance matrix in a space with a reduced number of chosen dimensions (Hair et al., 2009).

\section{Statistical Analyses}

Gene expression was compared between control and inoculated samples at times 0 and $3 \mathrm{~h}$ after inoculation using 3 statistical methods (Table 1). The version of the gene model used for this study was Ensembl Gene 92 (Aken et al., 2016). Three different tools were used, and these were chosen to study distinct and nonoverlapping methodologies.

Edge $R$ Tool. The method implemented in the edgeR package (Galaxy tool version 0.0.2; Robinson et al., 2010a) uses the quantile-adjusted conditional maximum likelihood procedure (Robinson and Smyth, 2008) in an exact test similar to the Fisher exact test to determine differential gene expression for pairwise comparisons. It assumes a negative binomial (NB) distribution for the data in a balanced incomplete block design as follows:

Table 1. Description of the statistical assumptions used in the methods for RNA sequencing differential gene expression analysis

\begin{tabular}{llll}
\hline Method $^{1}$ & Normalization $^{2}$ & Distribution & Differential expression test \\
\hline edgeR & TMM & Negative binomial & Exact test \\
baySeq & RLE & Negative binomial & $\begin{array}{l}\text { Posterior probabilities } \\
\text { Cuffdiff } 2\end{array}$ \\
\hline
\end{tabular}

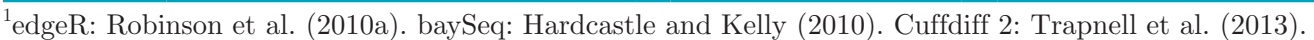

${ }^{2} \mathrm{TMM}=$ trimmed mean of $M$-values; RLE $=$ relative log expression; FPKM $=$ fragments per kilobase of transcript per million fragments sequenced. 


$$
Y_{g i} \sim \mathrm{NB}\left(M_{i} p_{g j}, \phi_{g}\right)
$$

where $g$ is the gene and $i$ is the sample, $M_{i}$ is the library size (total number of reads), $\phi_{g}$ is the dispersion, and $p_{g j}$ is the relative abundance of the gene $g$ in the experimental group $j$ to which the sample $i$ belongs. The NB parameterization where the mean was $\mu_{q i}=M_{i} p_{g j}$ and the variance was $\mu_{g i}\left(1+\mu_{g i} \phi_{g}\right)$ was used. The normalization factor named trimmed mean of $M$-values was estimated between each pair of samples (Robinson and Oshlack, 2010b). Individual gene expression was calculated as the mean expression of each gene averaged over all samples of each group and presented as the logarithm of counts per million reads. The BenjaminiHochberg procedure, proposed by Benjamini and Hochberg (1995), was used to control the false discovery rate (FDR), and a cut-off criterion of FDR-adjusted $P$ $<0.01$ was applied to identify differentially expressed genes.

BaySeq Tool. The method applied in the baySeq R/ Bioconductor package (version 1.16.0; Hardcastle and Kelly, 2010) uses prior information to fit a nonlinear model that quantifies biases and uncertainties in the estimates (Liu and Logvinenko, 2003). It also assumes an NB distribution for the data and derives an empirically determined prior distribution from the entire data set by maximum likelihood (Hardcastle and Kelly, 2010), represented as follows:

$$
Y_{i j} \sim \mathrm{NB}\left(M_{q l j}, \phi_{q}\right)
$$

where $Y_{i j}$ is the number of reads for the gene $i$ in the $j$ th sample, $M_{q l j}$ is the mean, and $\phi_{q}$ is the dispersion. The counting data from the data set with $\mathrm{n}=16$ samples $A$ $=\left\{A_{1}, \ldots, A_{16}\right\}$ are given by $\left(u_{i c}, \ldots, u_{n c}\right)$, where $u_{i c}$ is the counting for a given gene $c$ in sample $i$. For each sample $A_{i}$, the library size was obtained from a scale factor $l_{i}$. Thus, for each gene $(c)$, the data $(D)$ were considered as follows:

$$
D_{c}=\left[\left(u_{i c}, \ldots, u_{n c}\right),\left(l_{1}, \ldots, l_{16}\right)\right] .
$$

Two models were defined. In the first model, the entire data set, inoculated $(I)$ and control $(C)$, was considered as a single treatment:

$$
\begin{aligned}
D_{P C}= & {\left[\left(u_{I 1}, u_{I 2}, u_{I 3}, u_{I 4}, u_{C 1}, u_{C 2}, u_{C 3}, u_{C 4}\right),\right.} \\
& \left.\left(l_{I 1}, l_{I 2}, l_{I 3}, l_{I 4}, l_{C 1}, l_{C 2}, l_{C 3}, l_{C 4}\right)\right],
\end{aligned}
$$

whereas in the alternative model, the differences between inoculated $(I)$ and control $(C)$ data sets were considered:

$$
\begin{gathered}
D_{P}=\left[\left(u_{I 1}, u_{I 2}, u_{I 3}, u_{I 4}\right),\left(l_{I 1}, l_{I 2}, l_{I 3}, l_{I 4}\right)\right] \\
D_{C}=\left[\left(u_{C 1}, u_{C 2}, u_{C 3}, u_{C 4}\right),\left(l_{C 1}, l_{C 2}, l_{C 3}, l_{C 4}\right)\right] .
\end{gathered}
$$

Last, a normalization factor named relative log expression, described by Anders and Huber (2010), was used to compare both models. Posterior probabilities and FDR for each gene were calculated instead of significance values. A cut-off criterion of posterior probability $<0.01$ was applied to identify differentially expressed genes.

Cuffdiff 2 Tool. The Cuffdiff 2 method (Trapnell et al., 2013), implemented in Cufflinks software (Trapnell et al., 2010), was used with default settings. It uses a model for read counts based on a $\beta$-NB distribution and a $t$-test to identify differentially expressed genes and allows differential gene expression analysis between treatment and control based on a pairwise analysis without replicates. A geometric normalization procedure, classified by fragments per kilobase of transcript per million fragments sequenced, was applied to determine the minimum number of reads needed to reconstruct the entire sequence. Assumptions are

$$
x_{t}^{j} \sim \mathrm{BNB}\left(\mu_{t}^{j}, \sigma_{t}^{j^{2}}\right),
$$

where $x_{t}^{j}$ is the number of fragments in the replicate $j$ from the transcript $t$, BNB is the $\beta-\mathrm{NB}, \mu$ is the mean, and $\sigma^{2}$ is the variance. The Benjamini-Hochberg procedure was used as previously described in the baySeq method section.

\section{Identification of Metabolic Pathways}

The differentially expressed genes identified by each of the 3 methods were analyzed independently using DAVID Bioinformatic Resources 6.8 (Huang et al., 2009a,b), and pathways with $P<0.05$ were considered enriched.

\section{RESULTS AND DISCUSSION}

The total and the average number of reads generated by the RNA-seq were $842,604,108$ and $26,331,378$, respectively. The quality control performed using FastQC provided an overview of the variation in base quality scores at each position of the input file. The samples were of good quality based on the Phred scores (probability of incorrect base call).

Given the good quality of the cDNA libraries and the fact that mapping algorithms use only reads that perfectly match a position on the reference genome, the analyses were carried out without trimming the reads. On average, 88.91 (Table 2 ) and $88.89 \%$ of the 
Table 2. Total and proportion of aligned reads per sample

\begin{tabular}{|c|c|c|c|c|c|c|}
\hline \multirow{2}{*}{$\begin{array}{l}\text { Treatment and } \\
\text { sample label }^{1}\end{array}$} & \multicolumn{2}{|c|}{ Left reads } & \multicolumn{2}{|c|}{ Right reads } & \multicolumn{2}{|c|}{ Aligned pairs } \\
\hline & Total no. & $\%$ & Total no. & $\%$ & Total no. & $\%$ \\
\hline \multicolumn{7}{|l|}{ Control } \\
\hline G7T0 & $30,692,458$ & 89.9 & $30,419,535$ & 89.1 & $29,164,959$ & 84.2 \\
\hline G7T3 & $23,756,098$ & 87.9 & $23,934,459$ & 88.6 & $22,638,978$ & 82.6 \\
\hline G8T0 & $21,164,058$ & 90.4 & $21,137,250$ & 90.3 & $20,248,224$ & 85.3 \\
\hline G8T3 & $23,096,338$ & 89.0 & $23,253,768$ & 89.6 & $21,943,088$ & 83.9 \\
\hline G9T0 & $23,054,518$ & 87.5 & $23,235,820$ & 88.2 & $21,811,906$ & 82.3 \\
\hline G9T3 & $22,784,883$ & 90.2 & $22,690,679$ & 89.9 & $21,607,226$ & 85.0 \\
\hline G10T0 & $21,290,821$ & 88.6 & $21,216,345$ & 88.3 & $20,123,296$ & 82.6 \\
\hline G10T3 & $19,639,786$ & 89.2 & $19,736,210$ & 89.7 & $18,642,344$ & 83.9 \\
\hline \multicolumn{7}{|l|}{ Inoculated } \\
\hline G7T0 & $22,510,799$ & 86.3 & $22,658,924$ & 86.9 & $21,280,138$ & 80.9 \\
\hline G7T3 & $23,227,834$ & 89.8 & $23,142,353$ & 89.5 & $22,129,511$ & 84.2 \\
\hline G8T0 & $21,707,055$ & 88.3 & $21,809,049$ & 88.7 & $20,653,152$ & 82.8 \\
\hline G8T3 & $22,543,993$ & 89.5 & $22,395,590$ & 88.9 & $21,408,569$ & 83.8 \\
\hline G9T0 & $33,428,420$ & 90.4 & $33,434,970$ & 90.4 & $31,943,198$ & 85.1 \\
\hline G9T3 & $22,334,160$ & 88.4 & $22,479,819$ & 89.0 & $21,294,357$ & 83.2 \\
\hline G10T0 & $21,647,272$ & 86.5 & $21,777,734$ & 87.0 & $20,470,883$ & 80.5 \\
\hline G10T3 & $21,589,981$ & 89.5 & $21,473,579$ & 89.0 & $20,466,780$ & 83.8 \\
\hline
\end{tabular}

${ }^{1}$ G7 to G10: sample identification; T0 and T3: time (h) after inoculation.

trimmed and nontrimmed reads, respectively, were mapped to the reference genome. This difference is due to the lower number of reads in the trimmed data set. Similar results were reported by Cui et al. (2014), who also aligned sequenced mammary tissue samples using TopHat.

The unmapped reads subjected to the BLAST search were aligned to other members of the Bovidae family, and several of these regions are related to immune system processes, suggesting that the alleles are highly polymorphic and have not yet been annotated in the Bos taurus genome. During the multidimensional scaling analysis, the control sample G7T3 was clustered together with the inoculated samples (Figure 1), and it was removed from further analysis.

There are 26,453 annotated genes in the UMD 3.1 bovine genome assembly. A total of 27,404 sequences were expressed in the mammary tissue, of which 21,691 were annotated genes. The remaining 5,821 sequences were labeled as XLOC. A larger number of differentially expressed genes was reported by Cuffdiff 2 , followed by edgeR and baySeq (Table 3 ).

The variation in the number of differentially expressed genes is explained by the different statistical assumptions that each method is based on (Table 1). The different normalization methods that are used for these analyses may be largely responsible for the variability in the results, having more of an effect on the detection of genes differentially expressed than the statistical test used (Bullard et al., 2010; Maza et al., 2013).

The fragments per kilobase of transcript per million fragments sequenced (FPKM) normalization method is associated with the greatest number of false dis- coveries compared with relative log expression and trimmed mean of $M$-values methods; this also occurs in the production of mean squared errors, so the FPKM normalization method may not be the most suitable for differential gene expression analyses (Maza et al., 2013). The trimmed mean of $M$-values normalization method is satisfactory for maintaining a reasonable false-positive rate without any loss of power (Dillies et al., 2013) and performs very similar to relative log

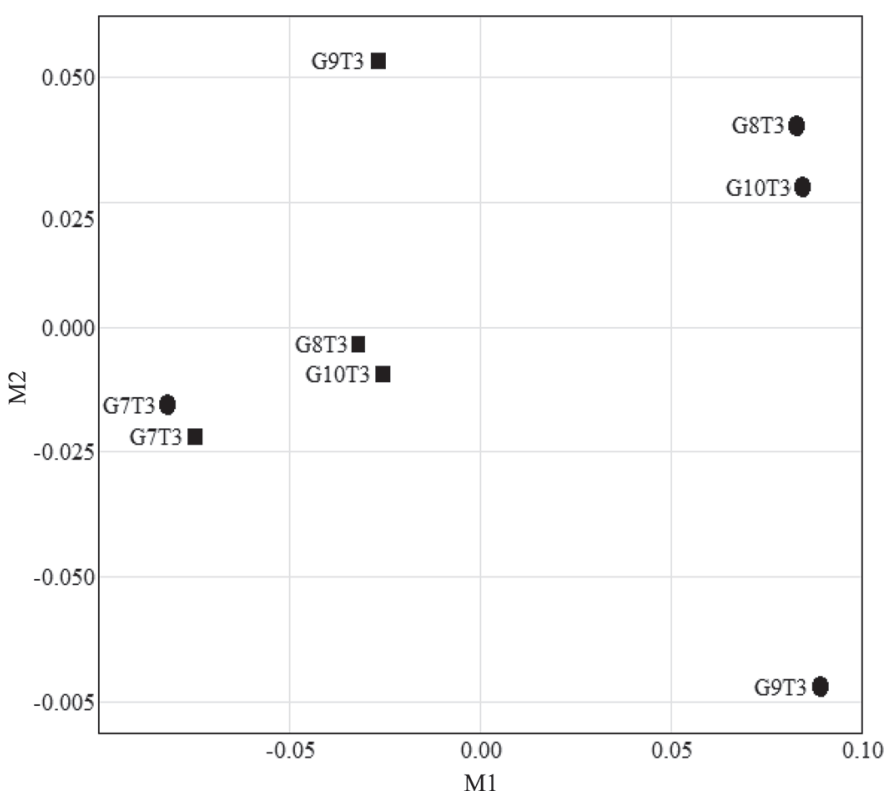

Figure 1. Multidimensional scaling plot of the samples in which distances correspond to normalized gene expression between pairs of samples. G7 to G10 = sample identification; T3 = time $3 \mathrm{~h}$ after inoculation; $\mathbf{\square}=$ inoculated; $\bullet=$ control. 
expression, once both can provide equal results in some simpler experiments (Maza et al., 2013; Maza, 2016). These methods perform well for the analysis of differentially expressed genes (Maza et al., 2013).

The baySeq assumes an NB distribution; however, it estimates posterior probabilities and the parameter distributions based on an empirical Bayesian method (Hardcastle and Kelly, 2010). Soneson and Delorenzi (2013) and Rapaport et al. (2013) observed that baySeq was computationally slower but had a better control of FDR with a large sample size and no outliers (Soneson and Delorenzi, 2013). In addition, baySeq it is known to be more conservative than the other methods (Rapaport et al., 2013; Soneson and Delorenzi, 2013; Seyednasrollah et al., 2015), which was corroborated in this study by the lower number of differentially expressed genes identified $(1,161)$.

In contrast, Cuffdiff 2 is considered less conservative and reported to have a higher FDR (Rapaport et al., 2013). The fact that the method is less conservative was also observed in the present study: 3,389 differentially expressed genes were identified by Cuffdiff 2 . According to Rapaport et al. (2013), Cuffdiff 2 uses a Poisson model to control variability in sequencing depth, biological noise, and splicing structure by calculating the mean count across replicates and $P$-values for any observed changes in a transcript's fragment count (Trapnell et al., 2013). The traditional statistical methods, as $t$-test, cannot be reliably applied to genomic data (Law et al., 2014). This method makes assumptions of normally distributed continuous data, whereas the reads are count based and cannot be normally distributed.

In the present study, the number of differentially expressed genes identified using edgeR was intermediary $(1,756)$ between the other 2 methods. The edgeR implements a frequentist method that uses an empirical Bayes procedure to adjust the overdispersion across genes by using the information of other genes (Robinson and Smyth, 2007, 2008). Soneson and Delorenzi (2013) considered the edgeR to be computationally efficient but less conservative compared with other methods, reporting a larger FDR. Rapaport et al. (2013) also reported variable FDR when comparing edgeR, baySeq, and Cuffdiff 2; however, both baySeq and edgeR had lower FDR.

The number of differentially expressed genes identified in the present study is in accordance with the literature because other studies have reported more genes detected by Cuffdiff 2, followed by edgeR and baySeq. The present results suggest that baySeq is more conservative than edgeR and Cuffdiff 2 and that the identification of differentially expressed genes by Cuffdiff 2 may be inflated (Table 3). An exception found in the literature, a study by Seyednasrollah et al. (2015), presented Cuffdiff 2 as conservative, reporting lower FDR compared with other methods.

Although the results from the 3 statistical tools were different (Figure 2), they seemed to be suitable for RNA-seq differential gene expression analysis in this study. There were only 122 shared genes among the methods. The most similar results were obtained between edgeR and baySeq, which shared 900 differentially expressed genes. In contrast, there were only 221 shared genes between Cuffdiff 2 and edgeR and only 145 shared genes between Cuffdiff 2 and baySeq.

A total of 5,519 transcripts were reported as differentially expressed, out of which 4,214 were annotated genes in UMD 3.1 genome assembly and were subjected to a pathway enrichment analysis (Figure 3). A total of 8,8 , and 64 pathways were significantly enriched $(P$ $<0.05$ ) for edgeR, baySeq, and Cuffdiff 2 , respectively. Table 4 presents the shared enriched pathways when studying the results from the 3 statistical methods. The platelet activation (bta04611) was the only pathway found to be enriched by all 3 methods, denoting that these methods resulted in different sets of differentially expressed genes. Four genes [Fc fragment of IgE receptor Ig (FCER1G), G protein subunit a i2 (GNAI2), ORAI calcium release-activated calcium modulator 1 (ORAI1), and vasodilator-stimulated phosphoprotein (VASP)] shared among the 3 methods studied were enriched in the pathway platelet activation; therefore, these genes should be indicated for future validation.

Using only the 122 shared differentially expressed genes among the 3 methods (Figure 2) could reduce the false-positive genes in the further analysis, supported by evidence of true differential expression in the tissue.

Table 3. Total and shared differentially expressed genes for the statistical contrasts according to the method used

\begin{tabular}{lcccc}
\hline & \multicolumn{3}{c}{ Method $^{2}$} & Shared \\
\cline { 2 - 3 } Contrast $^{1}$ & edgeR & baySeq & Cuffdiff 2 & genes (no.) \\
\hline Inoculated T0 $\times$ control T0 & 0 & 0 & 0 & 0 \\
Inoculated T3 $\times$ control T3 & 1,756 & 1,161 & 3,389 & 122 \\
\hline${ }^{1}$ T0 and T3: at time 0 and 3 h after inoculation, respectively. & & \\
${ }^{2}$ edgeR: Robinson et al. (2010a). baySeq: Hardcastle and Kelly (2010). Cuffdiff 2: Trapnell et al. (2013).
\end{tabular}


Table 4. Shared enriched pathways $(P<0.05)$ associated with the differentially expressed gene identified by 3 statistical methods ${ }^{1}$

\begin{tabular}{lll}
\hline edgeR and baySeq & edgeR and Cuffdiff 2 & baySeq and Cuffdiff 2 \\
\hline bta05012: Parkinson's disease & bta04611: platelet activation & bta04550: signaling pathways regulating pluripotency of \\
bta04611: platelet activation & bta04310: Wnt signaling pathway & stem cells \\
& bta05323: rheumatoid arthritis & bta04360: axon guidance \\
& bta04610: complement and coagulation & bta04611: platelet activation \\
& cascades & \\
\hline
\end{tabular}

edgeR: Robinson et al. (2010a). baySeq: Hardcastle and Kelly (2010). Cuffdiff 2: Trapnell et al. (2013).

However, the genes identified by one method or another are not always false positives because the experimental design can affect the effectiveness of the methods. Such approach would reduce the number of genes and the statistical power of the following enrichment analysis. Thus, using only the shared differentially expressed genes is not of interest for functional enrichment analysis. Instead, the shared genes could be used for validation by real-time PCR.

\section{CONCLUSIONS}

The results from the evaluated tools were different given their specific statistical assumptions. The use of the 3 methods for the detection of differentially ex-

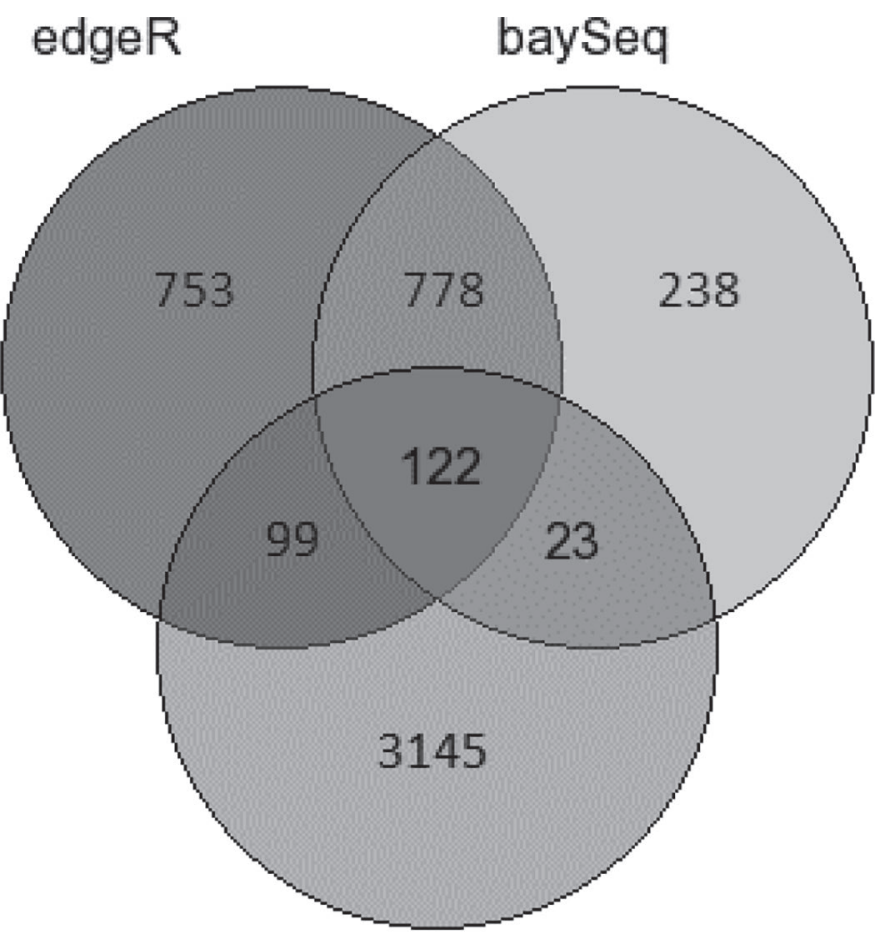

Cuffdiff 2

Figure 2. Venn diagram of the shared differentially expressed genes based on RNA seqencing data through 3 statistical methods: edgeR (Robinson et al., 2010a), baySeq (Hardcastle and Kelly, 2010), and Cuffdiff 2 (Trapnell et al., 2013). pressed genes helped select the genes to be indicated for validation. From the genes identified by the overlapping of the 3 methods, only platelet activation presented a significantly enriched pathway. We indicated the FCER1G, GNAI2, ORAI1, and VASP genes shared among the 3 methods in this pathway for posterior biological validation.

\section{ACKNOWLEDGMENTS}

The authors acknowledge the Brazilian Agricultural Research Corporation-Embrapa Dairy Cattle (Juiz de Fora, Minas Gerais, Brazil) for providing the data used in this study and the Bioinformatics Multiuser Laboratory (LMB) - Embrapa Agricultural Informatics

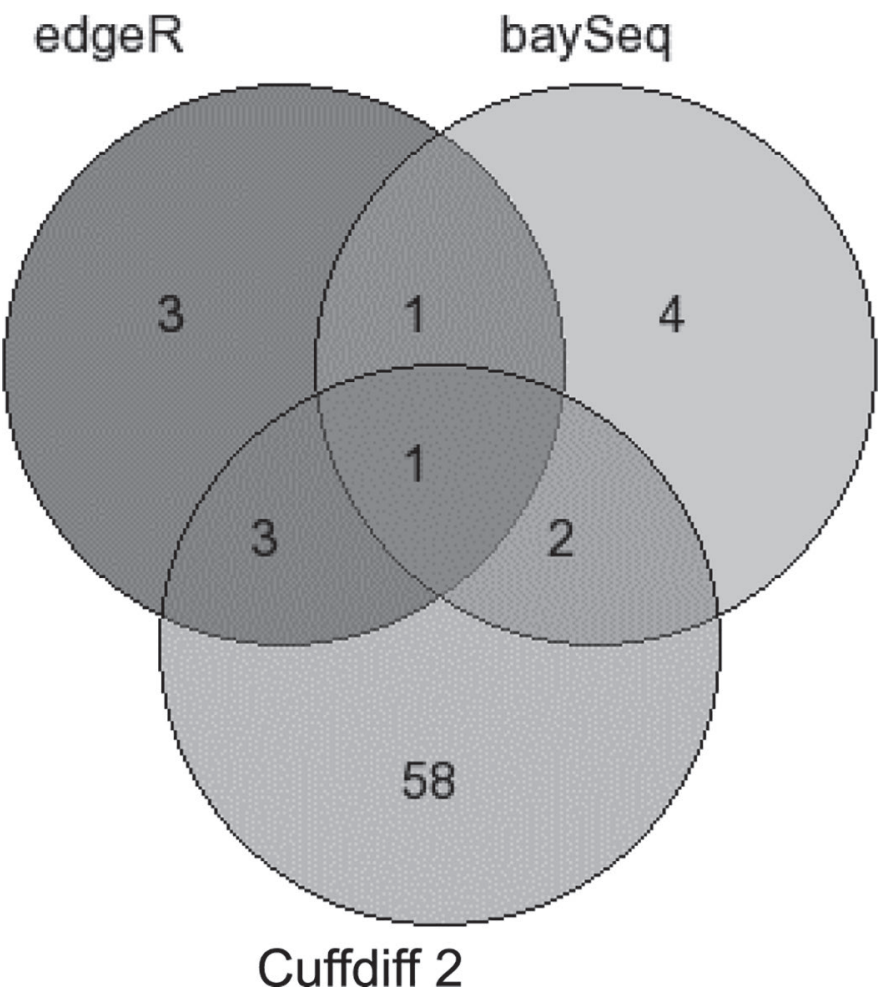

Figure 3. Venn diagram of the total number of significantly enriched pathways $(P<0.05)$ through 3 statistical methods: edgeR (Robinson et al., 2010a), baySeq (Hardcastle and Kelly, 2010), and Cuffdiff 2 (Trapnell et al., 2013). 
(Campinas, São Paulo, Brazil) for providing computational structure for data analysis. The first author received a Coordination for the Improvement of Higher Education Personnel (Coordenação de Aperfeiçoamento de Pessoal de Nível Superior, CAPES, Brazil)/ Embrapa scholarship. The experiment was financially supported by the Foundation for Research Support of the State of Minas Gerais (Fundação de Amparo à Pesquisa do Estado de Minas Gerais, FAPEMIG, Minas Gerais, Brazil; project number APQ-00095-15) and the National Council for Scientific and Technological Development (Conselho Nacional de Desenvolvimento Científico e Tecnológico, CNPq, Brazil; project number 473414/2011-2). Priscila Arrigucci Bernardes received a scholarship from the São Paulo Research Foundation (Fundação de Amparo à Pesquisa do Estado de São Paulo-FAPESP, São Paulo, Brazil; process numbers 2016/22940-3 and 2015/25096-6).

\section{REFERENCES}

Afgan, E., D. Baker, M. Van Den Beek, D. Blankenberg, D. Bouvier, M. Čech, J. Chilton, D. Clements, N. Coraor, C. Eberhard, B. Grüning, A. Guerler, J. Hillman-Jackson, G. Von Kuster, E. Rasche, N. Soranzo, N. Turaga, J. Taylor, A. Nekrutenko, and A. Goecks. 2016. The Galaxy platform for accessible, reproducible and collaborative biomedical analyses: 2016 update. Nucleic Acids Res. 44:W3-W10. https://doi.org/10.1093/nar/gkw343.

Aken, B. L., S. Ayling, D. Barrell, L. Clarke, V. Curwen, S. Fairley, J. F. Banet, K. Billis, C. G. Giron, T. Hourlier, K. Howe, A. Kahari, F. Kokocinski, F. J. Martin, D. N. Murphy, R. Nag, M. Ruffier, M. Schuster, Y. A. Tang, J.-H. Vogel, S. White, A. Zadissa, P. Flicek, and S. M. J. Searle. 2016. The Ensembl gene annotation system. Database (Oxford) 2016:1-19. https://doi.org/10.1093/database/ baw093.

Anders, S., P. T. Pyl, and W. Huber. 2015. HTSeq-A Python framework to work with high-throughput sequencing data. Bioinformatics 31:166-169.

Anders, S., and W. Huber. 2010. Differential expression analysis for sequence count data. Genome Biol. 11:R106.

Benjamini, Y., and Y. Hochberg. 1995. Controlling the false discovery rate: A practical and powerful approach to multiple testing. J. R. Stat. Soc. Series B Stat. Methodol. 57:289-300.

Bullard, J. H., E. Purdom, K. D. Hansen, and S. Dudoit. 2010. Evaluation of statistical methods for normalization and differential expression in mRNA-Seq experiments. BMC Bioinformatics 11:94. https://doi.org/10.1186/1471-2105-11-94.

Camacho, C., G. Coulouris, V. Avagyan, N. Ma, J. Papadopoulos, K. Bealer, and T. L. Madden. 2009. BLAST+: Architecture and applications. BMC Bioinformatics 10:421-429. https://doi.org/10 $.1186 / 1471-2105-10-421$.

Cui, X., Y. Hou, S. Yang, Y. Xie, S. Zhang, Y. Zhang, Q. Zhang, X. Lu, G. E. Liu, and D. Sun. 2014. Transcriptional profiling of mammary gland in Holstein cows with extremely different milk protein and fat percentage using RNA sequencing. BMC Genomics 15:226.

Dillies, M.-A., A. Rau, J. Aubert, C. Hennequet-Antier, M. Jeanmougin, N. Servant, C. Keime, G. Marot, D. Castel, J. Estelle, G. Guernec, B. Jagla, L. Jouneau, D. Laloe, C. L. Gall, B. Schaeffer, S. L. Crom, M. Guedj, and F. Jaffrézic.French StatOmique Con- sortium. 2013. A comprehensive evaluation of normalization methods for Illumina high-throughput RNA sequencing data analysis. Brief. Bioinform. 14:671-683. https://doi.org/10.1093/bib/bbs046.

Hair, J. F., W. C. Black, B. J. Badin, R. E. Anderson, and R. L. Tatham. 2009. Análise Multivariada de Dados. 6th ed. Artmed Editora S. A., Porto Alegre, RS, Brazil.

Hardcastle, T. J., and K. A. Kelly. 2010. baySeq: Empirical Bayesian methods for identifying differential expression in sequence count data. BMC Bioinformatics 11:422.

Huang, W., B. T. Sherman, and R. A. Lempicki. 2009a. Systematic and integrative analysis of large gene lists using DAVID Bioinformatics Resources. Nat. Protoc. 4:44-57. https://doi.org/10.1038/ nprot.2008.211.

Huang, W., B. T. Sherman, and R. A. Lempicki. 2009b. Bioinformatics enrichment tools: Paths toward the comprehensive functional analysis of large gene lists. Nucleic Acids Res. 37:1-13. https://doi .org/10.1093/nar/gkn923.

Law, C. W., Y. Chen, W. Shi, and G. K. Smyth. 2014. Voom: Precision weights unlock linear model analysis tools for RNA-seq read counts. Genome Biol. 15:R29.

Liu, J. S., and T. Logvinenko. 2003. Bayesian methods in biological sequence analysis. Pages 66-93 in Handbook of Statistical Genetics. 2nd ed. Wiley, Chichester, UK.

Maza, E. 2016. In papyro comparison of TMM (edgeR), RLE (DESeq2), and MRN normalization methods for a simple two-conditions-without-replicates RNA-Seq experimental design. Front. Genet. 7:164. https://doi.org/10.3389/fgene.2016.00164.

Maza, E., P. Frasse, P. Senin, M. Bouzayen, and M. Zouine. 2013. Comparison of normalization methods for differential gene expression analysis in RNA-Seq experiments. Commun. Integr. Biol. 6:e25849. https://doi.org/10.4161/cib.25849.

Rapaport, F., R. Khanin, Y. Liang, M. Pirum, A. Krek, P. Zumbo, C. E. Mason, N. D. Socci, and D. Betel. 2013. Comprehensive evaluation of differential gene expression analysis methods for RNA-seq data. Genome Biol. 14:R95.

Robinson, M. D., D. J. McCarthy, and G. K. Smyth. 2010a. edgeR: A Bioconductor package for differential expression analysis of digital gene expression data. Bioinformatics 26:139-140.

Robinson, M. D., and A. Oshlack. 2010b. A scaling normalization method for differential expression analysis of RNA-seq data. Genome Biol. 11:R25.

Robinson, M. D., and G. K. Smyth. 2007. Moderated statistical tests for assessing differences in tag abundance. Bioinformatics 23:28812887.

Robinson, M. D., and G. K. Smyth. 2008. Small-sample estimation of negative binomial dispersion, with applications to SAGE data. Biostatistics 9:321-332.

Seyednasrollah, F., A. Laiho, and L. L. Elo. 2015. Comparison of software packages for detecting differential expression in RNA-seq studies. Brief. Bioinform. 16:59-70. https://doi.org/10.1093/bib/ bbt086.

Soneson, C., and M. Delorenzi. 2013. A comparison of methods for differential expression analysis of RNA-seq data. BMC Bioinformatics 14:91.

Trapnell, C., D. G. Hendrickson, M. Sauvageau, L. Goff, J. L. Rinn, and L. Pachter. 2013. Differential analysis of gene regulation at transcript resolution with RNA-seq. Nat. Biotechnol. 31:46-53.

Trapnell, C., L. Pachter, and S. L. Salzberg. 2009. TopHat: Discovering splice junctions with RNA-Seq. Bioinformatics 25:1105-1111.

Trapnell, C., B. A. Williams, G. Pertea, A. Mortazavi, G. Kwan, M. J. V. Baren, S. L. Salzberg, B. J. Wold, and L. Pachter. 2010. Transcript assembly and quantification by RNA-Seq reveals unannotated transcripts and isoform switching during cell differentiation. Nat. Biotechnol. 28:511-515.

Wang, Z., M. Gerstein, and M. Snyder. 2009. RNA-Seq: A revolutionary tool for transcriptomics. Nat. Rev. Genet. 10:57-63. 